Da wir hier überhaupt nur speziellere Aussagen bei Voraussetzung einer Resonanzstelle machen können, bleiben bei mehreren, bei $\mathrm{Hg}$ evtl. auf verschiedene Isotope verteilten Resonanzstellen noch sehr viele Möglichkeiten für ihre Energien und Breiten.*

Bei Gadolinium und Europium hängt endlich wegen ihrer größeren T.E.-Werte der Exponent des Abfallgesetzes stärker von der in Abb. 2 dargestellten Maxwell-Korrektur ab. Bei Zugrundelegung der Korrekturen von Abb. 2 erhält man für Gd und Eu wahre T.E.-Werte von 1,49 bzw. 1,56 und die zugehörigen Exponenten $\varphi=0,85 \mathrm{bzw}$. 0,95. Dann ergibt sich aus Abb.1, daß bei beiden Elementen $E_{\mathrm{r}}$ unterhalb $k T$, aber unabhängig von $\Gamma$ oberhalb $-0,13 \mathrm{eV}(\varepsilon=-4)$ liegen muß, und daß $\Gamma$ kleiner als etwa $0,15 \mathrm{eV}$ ist. Für $\mathrm{Eu}$ sind natürlich die Einschränkungen noch etwas schärfer. Da Eu nur zwei, Gd aber,5 etwa gleich häufige Isotope besitzt, der Einfangquerschnitt von Gd aber trotzdem den des Eu um fast einen Faktor 10 übertrifft, muß wohl nach Gl. (1) das

* Die Messungen an Quecksilber mit dem Columbiaund Clinton-Spektrometer ergeben keinen stärkeren Abfall im thermischen Gebiet als 1/v. Eine Resonanzstelle bei $-2,0 \mathrm{eV}$, auf die aus dem Gesamtverlauf bis $10 \mathrm{eV}$ geschlossen wird, hätte sich in den vorliegenden Messungen nicht bemerkbar machen können.
Verhältnis von $\Gamma_{\mathbf{N}}$ zu $\Gamma$ (Neutronenbreite $\mathrm{zu}$ Gesamtbreite des Niveaus) für Gd wesentlich größer sein als für $\mathrm{Eu}$, selbst wenn man von der innerhalb der eben abgeleiteten Grenzen noch bestehenden Möglichkeiten Gebrauch macht, die Resonanzstelle des Gd möglichst nahe bei $k T$ und die des Eu nahe bei $-0,13 \mathrm{eV}$ anzunehmen.**

Die hier beschriebenen Versuche sind im KaiserWilhelm-Institut für Physik in Berlin-Dahlem 1942 bis 1943 durchgeführt worden; sie konnten aus äußeren Gründen bishèr nicht veröffentlicht werden. Ich halte die späte Veröffentlichung dieser Messungen trotz der außerordentlichen Fortschritte, die inzwischen in Amerika in der Neutronenspektrometrie erzielt wurden, für gerechtfertigt, weil die hier angewandte Methode sich durch die Ausschaltung der Streuung von den amerikanischen Durchlässigkeitsmessungen unterscheidet. Um den Zusammenhang der Arbeit, die bei Kriegsende abgeschlossen wurde, nicht zu unterbrechen, habe ich auf die Ergebnisse der amerikanischen Spektrometerarbeiten in Fußnoten hingewiesen.

** Auch die Untersuchungen an Gadolinium und Europium im Argonne-Laboratorium liefern einen steileren Abfall als' $1 / v$ für Gadolinium bei $0,03 \mathrm{eV}$ und einen fast exakten $1 / v^{2}$-Abfall für Europium. Bei Gadolinium scheint die Hauptresonanz gerade bei etwa $0,03 \mathrm{eV}$ zu liegen, was zu einer stärkeren Krümmung der genauen Absorptionskurve an dieser Stelle führt. Europium hat Resonanzstellen bei $0,46 \mathrm{eV}$ und höheren Energien und die bei meinen thermischen Messungen allein maßgebende Resonanz unterhalb $0,03 \mathrm{eV}$, deren Lage nicht genau bestimmt ist.

\title{
Beziehungen zwischen der Kernladungszahl, effektiven Kernladungszahl und Verbrennungswärme
}

\author{
Von Benno LenHard* \\ (Z. Naturforschg. 4 a, 257-261 [1949]; eingegangen am 22. Juli 1948)
}

\begin{abstract}
Angabe einer Näherungsformel für die Berechnung der effektiven Kernladungszahlen aus' den Kernladungszahlen und Hauptquantenzahlen und einer Formel für die Berechnung der Verbrennungswärmen der Kohlenwasserstoffe aus den Kernladungszahlen.
\end{abstract}

\section{$\S 1$. Die effektive Kernladung}

$\mathrm{B}$ ei empirisch bekannter Ionisierungsenergie $E$ kann man die effektive Kernladung eines neutralen Atoms berechnen aus:

$$
Z_{\text {eff }}=\bar{n} \sqrt{E / \overline{R h}}
$$

wo $R=$ Rydberg-Frequenz, $h=$ Plancksches Wirkungsquantum und $\bar{n}=$ Hauptquantenzahl des

* Z. Zt. (13a) Kulmbach, Fischergasse 13. äußersten Elektrons im Grundzustand, also z. B. bei Na $\bar{n}=3$. Verf. fand nun, daß für die Alkaliatome, bei welchen nur ein $s$-Elektron, und für die Elemente $\mathrm{B}, \mathrm{Al}, \mathrm{Ga}$, In und $\mathrm{Tl}$, bei welchen nur ein $p$-Elektron Valenzelektron ist, in erster Annäherung gesetzt werden kann:

$$
Z_{\mathrm{eff}}=\sqrt{Z_{i}}
$$

wie Tab. 1 zeigt: 


\begin{tabular}{|c|c|c|c|c|c|c|c|c|c|c|c|c|}
\hline & $\mathrm{H}$ & $\mathrm{Li}$ & B & $\mathrm{Na}$ & $\cdot \mathrm{Al}$ & $\mathrm{K}$ & $\mathrm{Ga}$ & $\mathrm{Rb}$ & In & $\mathrm{Cs}$ & $\mathrm{Tl}$ & - \\
\hline$Z=$ & 1 & 3 & 5 & 11 & 13 & 19 & 31 & 37 & 49 & 55 & 81 & 87 \\
\hline $\bar{n}=$ & 1 & 2 & 2 & 3 & 3 & 4 & 4 & 5 & 5 & 6 & 6 & 7 \\
\hline$Z_{\text {eff }}$ nach Gl. $(1)=$ & 1 & 1,26 & 1,56 & 1,84 & 1,98 & 2,26 & 2,66 & 2,78 & 3,25 & 3,20 & 4,0 & $(3,78)$ \\
\hline$Z_{\text {eff }}$ nach Gl. (2) $=$ & 1 & 1,23 & 1,58 & 1,91 & 2,08 & 2,18 & 2,78 & 2,72 & 3,12 & 3,03 & 3,67 & 3,53 \\
\hline $\begin{array}{l}\text { Abweichung (2) } \\
\quad \text { von (1) in } \%=\end{array}$ & 0 & $-2,5$ & $+1,4$ & $+3,5$ & $+5,0$ & $-3,5$ & $+4,5$ & $-2,0$ & $-4,0$ & $-5,5$ & $-8,0$ & $(-7,0)$ \\
\hline
\end{tabular}

Tab. 1.



Abb.1. Gemessene und berechnete Ionisierungsenergien aller Elemente. — empirische Werte; ...... nach Gl. (3) berechnete Werte.

Die größte Abweichung des Wertes aus Gl. (2) vom empirischen $Z_{\text {eff }}$-Wert ist bei $\mathrm{Tl}-8,0 \%$, die mittlere Abweichung für alle 12 Elemente nur $-1,5 \%$.
Bei Gleichsetzung der rechten Seiten der Gln. (1) und (2) erhält man:

$$
E=R h Z / \bar{n}^{3},
$$




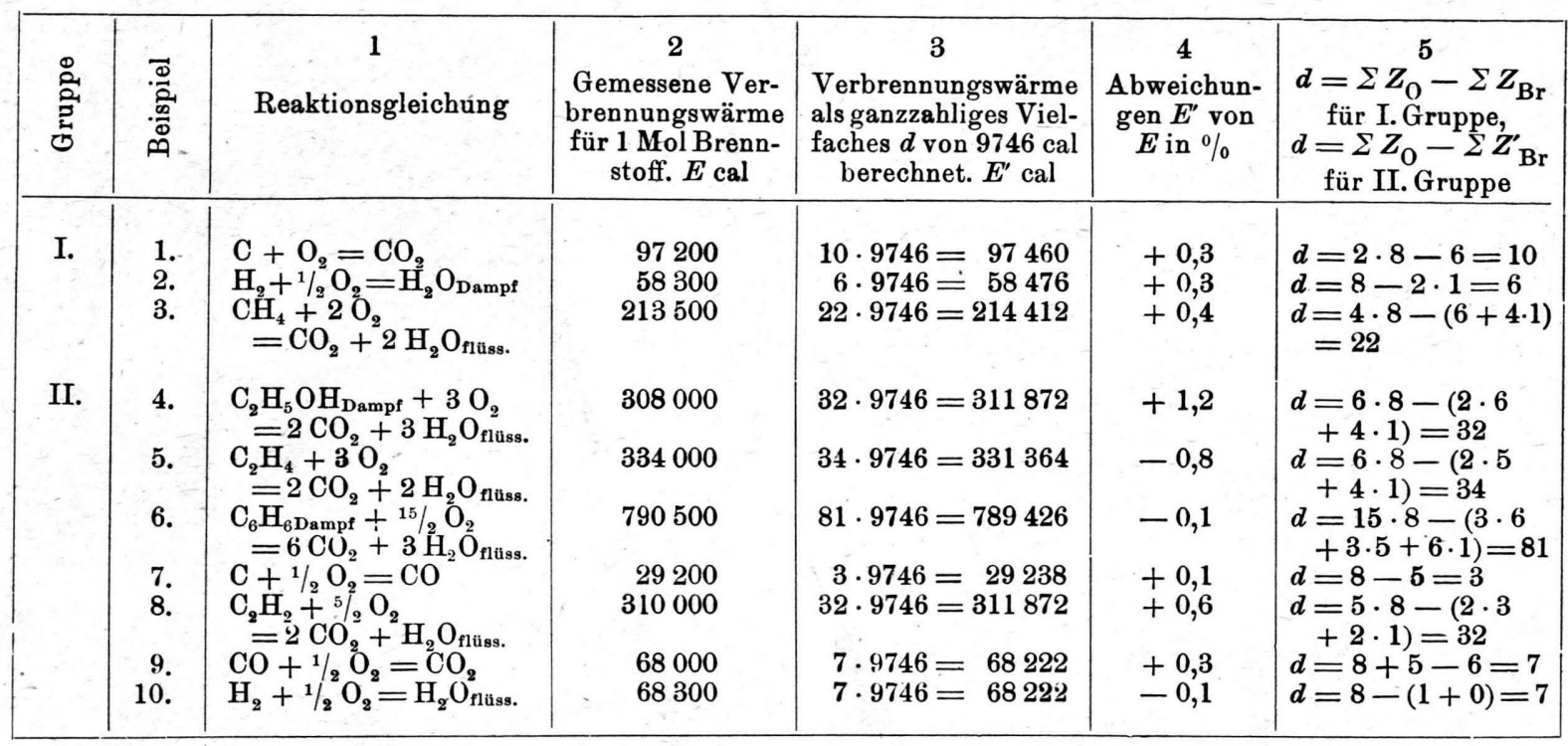

Tab. 2. Vergleich der gemessenen und berechneten Verbrennungswärmen von C, H und den .Kohlenwasserstoffverbindungen.

Empirische Werte der Spalte 2 für 2. Beispiel aus Staudingers Tabellen, 2. Aufl., 1935, S. 97; für 6. und 10. Beispiel aus A.Eucken, Grundriß der physikal. Chemie, 5. Aufl. [1942], S. 140; für die übrigen Beispiele aus Kalender für das Gas- und Wasserfach, herausgeg. vom Deutschen Verein von Gas- und Wasserfachmännern, Jahrg. 1927, S. 212.

welche Gleichung für die Ionisierungsenergien der 12 genannten Elemente gute Übereinstimmung mit den empirischen Werten gibt, wie aus der Abb. 1 ersichtlich ist.

Das Diagramm zeigt, daß auch für die Elemente mit den Ordnungszahlen $Z=8,57,72$ bis 79,89 bis 92 gute Ubereinstimmung zwischen den errechneten und gemessenen Ionisierungsenergien vorhanden ist, während für die übrigen Elemente vermutlich wegen der Coulomb-Abstoßung zwischen den Hüllelektronen mehr oder weniger große Abweichungen gegeben sind. Für die Edelgase mit großer Ordnungszahl ( $\mathrm{X}$ und $\mathrm{Rn}$ ) gilt mit großer Annäherung $E=2 R h Z / \bar{n}^{3}$.

§ 2. Die Verbrennungswärmen von Kohlenstoff, Wasserstoff und den Kohlenwasserstoffen

Verf. fand, daß man die Verbrennungswärmen von 1 Mol Kohlenstoff, Wasserstoff oder Kohlenwasserstoff durch die Gleichung $E=9746 \cdot d$ cal darstellen kann, wo $d$ eine ganze, für den betreffenden Brennstoff charakteristische Zahl ist. Tab. 2 möge dies beweisen.

Die Werte der 3. Spalte der Tab. 2 weichen meistens, wie aus der 4. Spalte ersichtlich ist, um weniger als $0,5 \%$ von den gemessenen der 2. Spalte ab, die Übereinstimmung ist also gut.

Es ist nun weiter möglich, die ganze Zahl $d$ bei den Beispielen der I. Gruppe als Differenz der Ordnungszahlen $Z$ der an der chemischen Reaktion beteiligten Elemente darzustellen. Ist nämlich $\Sigma Z_{\mathrm{Br}}$ die Summe der Ordnungszahlen aller Atome des Brennstoffmoleküls und $\Sigma Z_{0}$ die Summe der Ordnungszahlen aller bei der Reaktion zur Bindung kommenden O-Atome, so gilt für die I. Gruppe der Beispiele die einfache Beziehung:

$$
d=\Sigma Z_{0}-\Sigma Z_{\mathrm{Br}} \text {. }
$$

Die Berechnung der $d$-Werte ist für die einzelnen Beispiele der I. Gruppe aus der 5. Spalte ersichtlich.

Für die I. Gruppe der Reaktionen läßt sich somit die Verbrennungswärme für $1 \mathrm{Mol}$ Brennstoff nach der Gleichung berechnen:

$$
E=\left(\Sigma Z_{\mathrm{O}}-\Sigma Z_{\mathrm{Br}}\right) \cdot 9746 \mathrm{cal} .
$$

Folglich erhalten wir für die Verbrennungswärme von einem einzigen Brennstoffmolekül, indem wir durch die Loschmidtsche Konstante dividieren und die Verbrennungsenergie in Wattsec ausdrücken: 


$$
E=\left(\Sigma Z_{0}-\Sigma Z_{\mathrm{Br}}\right) \cdot 0,678 \cdot 10^{-19} \mathrm{~W} \text { attsec } .
$$

Es ist aber $0,678 \cdot 10^{-19}$ Wattsec $=21,7 \cdot 10^{-19}: 32$ Wattsec $=R h / 32$, wo $R=$ Rydberg-Frequenz und $h=$ Plancksches Wirkungsquantum ist. Wir gelangen so auf empirischem Wege zu der Formel für die Verbrennungsenergie von einem einzigen Brennstoffmolekül der I. Gruppe:

$$
E=\left(\Sigma Z_{0}-\Sigma Z_{\mathrm{Br}}\right) \cdot R h / 32 .
$$

Diese Formel hat nun nicht nur für. $\mathrm{C}, \mathrm{H}$ und $\mathrm{CH}_{4}$, sondern für alle gesättigten Kohlenwasserstoffe der Methanreihe $\mathrm{C}_{n} \mathrm{H}_{2 n+2}$ Gültigkeit. Zum Beweise greifen wir auf die Dulongsche Verbandsformel zurück. Nach dieser ist die Verbrennungswärme von Gasen, welche keine ungesättigten Kohlenwasserstoffe enthalten:

$$
E=8100 \mathrm{C}+29150(\mathrm{H}-\mathrm{O} / 8) \mathrm{cal},
$$

wo C der Gehalt an Kohlenstoff, $\mathrm{H}$ der an Wasserstoff und $\mathrm{O}$ der an Sauerstoff in Gramm ist. In $1 \mathrm{Mol}=16 \mathrm{~g} \mathrm{CH}_{4}$ sind also z. B. $12 \mathrm{~g} \mathrm{C}$ und $4 \mathrm{~g} \mathrm{H}$. Nach der Verbandsformel erhalten wir somit:

$$
\begin{aligned}
E & =8100 \cdot 12+29150 \cdot 4 \\
& =97200+58300 \cdot 2=213800 \mathrm{cal} .
\end{aligned}
$$

Die in der letzten Zeile vorkommenden Zahlenwerte sind aber genau die Verbrennungswärmen für je $1 \mathrm{Mol} \mathrm{C}, \mathrm{H}$ und $\mathrm{CH}_{4}$ der Tab. 2. Die Verbandsformel ist also nur die empirische Darstellungsform der Gl. (5), weshalb deren Gültigkeitsbereich der gleiche wie der der Verbandsformel ist.

Für die Beispiele der II. Gruppe der Tab. 2 hat die Gl. (5) und ihre empirische Formulierung in der Verbandsformel nur beschränkte Gültigkeit. So ist z. B. die gemessene Verbrennungswärme für flüssigen 100-proz. Äthylalkohol $6480 \mathrm{cal} / \mathrm{g}$ $=298080 \mathrm{cal} / \mathrm{Mol}$. Da die Verdampfungswärme des Alkohols 216,7 cal/g und sein Molekulargewicht 46 ist, ist die Verdampfungswärme von $216,7 \cdot 46=9970 \mathrm{cal} / \mathrm{Mol}$ noch zu addieren, um die Verbrennungswärme für 1 Mol Äthylalkoholdampf in Höhe von etwa 308000 cal zu erhalten. Dieser Wert ist für das vierte Beispiel in die 2. Spalte eingetragen. Nach der Verbandsformel erhalten wir:

$E=8100 \cdot 24+29150 \cdot(6-16 / 8)=311000 \mathrm{cal} / \mathrm{Mol}$.

Man nennt die Differenz $(\mathrm{H}-\mathrm{O} / 8)$ in der Verbandsformel den disponiblen Wasserstoffgehalt.
Es sind also nur $(6-16 / 8)=4$ disponible $\mathrm{H}$ Atome in einem $\mathrm{C}_{2} \mathrm{H}_{5} \mathrm{OH}-$ Molekül.

Bilden wir nun die Summe der Ordnungszahlen nur aus den Ordnungszahlen der zwei C-Atome und der vier disponiblen $\mathrm{H}$-Atome, so ist $\Sigma Z_{\mathrm{Br}}^{\prime}=2 \cdot 6+4 \cdot 1=16$. Weiter ist $\Sigma Z_{\mathrm{O}}=6 \cdot 8=48$ für die drei zur Bindung kommenden freien Sauerstoffmoleküle. Dann ist nach Gleichung (4) $d=\Sigma Z_{0}-\Sigma Z_{\mathrm{Br}}^{\prime}=48-16=32$, welcher Wert mit dem $d$-Wert der 3. Spalte der Tabelle übereinstimmt. Gl. (5) behält also beschränkte Gültigkeit, insofern $\Sigma Z_{\mathrm{Br}}^{\prime}$ an Stelle von $\Sigma Z_{\mathrm{Br}}$ gesetzt werden muß. Allgemein muß somit für die Sauerstoff enthaltenden Kohlenwasserstoffverbindungen an Stelle der Summe der Ordnungszahlen der HAtome nur die Summe der Ordnungszahlen der disponiblen $\mathrm{H}$-Atome in Gl. (5) eingesetzt werden.

In ähnlicher Weise kann Gl. (5) auch auf die ungesättigten Kohlenwasserstoffe durch Einführung von $Z^{\prime}$-Werten angewandt werden. So ist bei der Doppelbindung $\mathrm{C}=\mathrm{C}$ entweder für beide $\mathrm{C}$ Atome statt $Z=6$ nur $Z^{\prime}=5$ oder für das eine C-Atom $Z=6$, für das 2. C-Atom aber nur $Z^{\prime}=4$ in Gl. (5) einzusetzen, so daß für beide C-Atome $\Sigma Z^{\prime}=10$ ist. Auf diese Weise erhält Gl. (5) Gültigkeit für Äthylen (5. Beispiel), die homologe Olefinreihe $\mathrm{C}_{n} \mathrm{H}_{2 n}$ und für den Benzolring (6. Beispiel), bei welchem, den 3 einfachen und den 3 Doppelbindungen zwischen den C-Atomen entsprechend, für $3 \mathrm{C}$-Atome die Ordnungszahl $Z=6$, für $3 \mathrm{C}$-Atome aber $Z^{\prime}=5$ in Gl. (5) eingesetzt werden muß. Auch bei der Verbrennung von $\mathrm{C}$ zu CO (7. Beispiel) sind Bindungen der Art gegeben, daß in gleicher Weise wie bei der Doppelbindung $Z^{\prime}=5$ in Gl. (5) einzusetzen ist.

Bei der Dreifachbindung $\mathrm{C} \equiv \mathrm{C}$ im Acetylen (8. Beispiel) und der homologen Reihe $\mathrm{C}_{n} \mathrm{H}_{2 n-2}$ muß statt der Ordnungszahl $Z=6$ entweder für beide an der Dreifachbindung beteiligten C-Atome $Z^{\prime}=3$ oder für das eine C-Atom $Z=6$, für das zweite C-Atom aber $Z^{\prime}=0$ eingesetzt werden, so daß für beide C-Atome $\Sigma Z^{\prime}=6$ ist. Die auf diese Weise durchgeführten Berechnungen der $d$-Werte sind aus Tab. 2, Spalte 5, zu ersehen.

Zusammenfassend kann man also die Regel aufstellen, daß bei der Doppelbindung $\mathrm{C}=\mathrm{C}$ für das zweite Atom $Z^{\prime}=Z-Z_{\mathrm{K}}=6-2=4$, bei der Dreifachbindung $\mathrm{C} \equiv \mathrm{C}$ für das zweite Atom $Z^{\prime}=Z-\left(Z_{\mathrm{K}}+Z_{\mathrm{L}}\right)=6-(2+4)=0$ in Gl. (5) einzusetzen ist, wo $Z_{\mathrm{K}}$ die negative Ladung der $\mathrm{K}$-Schale und $Z_{\mathrm{L}}$ die der L-Schale des C-Atoms 
ist. Beim Benzolring gilt dann für die 3 Einfachbindungen $\Sigma Z_{1}=3 \cdot(Z+Z)=3 \cdot(6+6)=36$, für die 3 Doppelbindungen $\Sigma Z_{2}^{\prime}=3 \cdot\left[Z+\left(Z-Z_{\mathrm{K}}\right)\right]$ $=3 \cdot[6+(6-2)]=30$. Da hier jedes C-Atom doppelt in Rechnung gestellt ist, ergibt sich für die 6 C-Atome $\Sigma Z^{\prime}=\left(\Sigma Z_{1}+\Sigma Z_{2}^{\prime}\right): 2=33$.

Beim 9. Beispiel stellt sich die Verbrennungswärme nach dem Heßschen Satz als Differenz aus den Verbrennungswärmen des 1 . und 7. Beispiels dar. Es ist $d=8+5-6=7$, d.h. das ${ }^{-}$C-Atom geht von einem der Doppelbindung ähnlichen $\mathrm{Zu}$ stand zum Zustand der Einfachbindung über. Die Kernladungszahl des gebundenen O-Atoms bleibt in gleicher Weise wie beim 4 . Beispiel unberücksichtigt.

Beim Vergleich des 10. und 2. Beispiels erweist sich die Verdampfungswärme von $1 \mathrm{Mol} \mathrm{H}_{2} \mathrm{O}$ gleich 9746 cal, von einem $\mathrm{H}_{2} \mathrm{O}$-Molekül gleich $R h / 32$. Es muß hier für das zweite $\mathrm{H}$-Atom in analoger Weise wie bei der $\mathrm{C}^{*}=\mathrm{C}$-Bindung statt $Z=1$ nur $Z^{\prime}=Z-Z_{\mathrm{K}}=1-1=0$ gesetzt werden, wo $Z_{\mathrm{K}}$ die negative Ladung der K-Schale des H-Atoms ist.

Gerade diese Einschränkungen in der Anwendbarkeit der Gl. (5) bei den Beispielen der II. Gruppe gestatten, da auch bei diesen Einschränkungen eine Gesetzmäßigkeit zu erkennen ist, Rückschlüsse auf den Bindungscharakter zwischen den Atomen dieser Moleküle, worauf aber an dieser Stelle nicht weiter eingegangen werden soll. Soviel sei hier zur atomphysikalischen Deutung der Gl. (5) erwähnt: Zerlegen wir dieselbe in die beiden Glieder

und

$$
E_{1}=R h / 32 \cdot \Sigma Z_{0}
$$

$$
E_{2}=R h / 32 \cdot \Sigma Z_{\mathrm{Br}}
$$

so stellt $E_{1}$ die Elektronenaffinität bei der Anlagerung von Elektronen an die neutralen $\mathrm{O}$ Atome, also die bei der Bildung von Sauerstoffanionen freiwerdende Energie, $\boldsymbol{E}_{2}$ die bei der Abtrennung von Brennstoffvalenzelektronen aufzuwendende Ionisierungsenergie dar. Wir kommen somit zu einer sinnvollen Deutung der Differenz $E=E_{1}-E_{2}$, wenn wir annehmen, daß ein oder mehrere Valenzelektronen der Atome des Brennstoffmoleküls bei der Verbrennung vollständig zu den Sauerstoffatomen übertreten, also polare Bindungen herstellen, während die Atome im Brennstoffmolekül, soweit es sich um Beispiele der I. Gruppe handelt, vor der Verbrennung unter sich nur unpolar gebunden sind. Bei der Elektronenabtrennung von den Brennstoffatomen wird die Energie $E_{2}$ aufgewandt, beim Anbau an die neutralen O-Atome aus elektrostatischen Gründen die Energie $E_{1}$ gewonnen. Bei den Alkoholen ist dieser Energieüberschuß kleiner als bei den entsprechenden Kohlenwasserstoffen, weil bereits in diesen polare Bindungen zum gebundenen O-Atom bestehen, was rechnerisch dadurch zum Ausdruck kommt, daß nur die Ordnungszahlen der freien $\mathrm{O}$-Atome und der disponiblen $\mathrm{H}$-Atome in Gl. (5) eingesetzt werden dürfen.

Der analoge Bau der Gln. (3), (6) und (7), bei welchen sich die Ionisierungsenergie als der Kernladungszahl $Z$ proportional erweist, läßt den Zusammenhang zwischen diesen Gleichungen vermuten. In der Tat gibt Gl. (3), wie aus dem obigen Diagramm ersichtlich ist, sehr gute Werte für $\mathrm{H}$ und $\mathrm{O}$, einen etwas weniger guten für $\mathrm{C}$. Es läßt sich nun zeigen, daß Gl. (3) auch Gültigkeit für die angeregten Atomzustände besitzt, wenn man sie in die Form bringt:

$$
E=\frac{R h}{f^{2}(n, l)} \frac{Z}{\bar{n}},
$$

wo $f$ eine Funktion der Hauptquantenzahl $n$ des angeregten Zustands und der Nebenquantenzahl $l$ ist. Diese Betrachtungen sollen hier nicht weiter ausgeführt werden. Der Nenner 32 der Gln. (6) und (7) entspricht dann dem Nenner der Gl. (8), also

$$
32=f^{2}(n, l) \cdot \bar{n} .
$$

Aus der Größe 32 des Nenners ist zu ersehen, daß es sich bei der Verbrennung nicht um Elektronenübergänge von einem Grundzustand zum anderen handeln kann, sondern um Übergänge von angeregten Zuständen der Brennstoffatome zu angeregten Zuständen der O-Atome, wie sie im Molekülverband gegeben sind, oder doch während der Verbrennung kurzlebig auftreten können. Es läßt sich besonders zeigen, daß bei diesen Elektronenübergängen die Nebenquantenzahl der verlassenen Bahn des Brennstoffatoms immer mit der Nebenquantenzahl der erreichten Bahn des OAtoms übereinstimmt. 